\title{
Commentary on: Effects of mesh-related complications in vaginal surgery on quality of life
}

\author{
Abdelmageed Abdelrahman ${ }^{1}$ \\ Received: 2 July 2018 / Accepted: 28 December 2018 / Published online: 14 January 2019 \\ (C) The International Urogynecological Association 2019
}

This was a cross sectional study of 128 women who had vaginal mesh surgery in a Dutch university hospital between 2007 and 2012. The authors hypothesized that health-related quality of life (HR-QoL) is comparable in women after vaginal mesh surgery regardless of the presence or absence of a mesh-specific complication. HR-QoL was defined as being related to micturition, defecation, and sexual function. This was assessed using the Dutch versions of the following validated questionnaires: Urogenital Distress Inventory short form (UDI-6), Incontinence Impact Questionnaire (IIQ), Defecation Distress Inventory (DDI), and Pelvic Organ Prolapse/Urinary Incontinence Sexual Function Questionnaire short form (PISQ-12). Indications to perform vaginal mesh surgery were recurrence of pelvic organ prolapse (POP), women with a posterior vaginal wall prolapse after previous vaginal hysterectomy, or women participating in studies to evaluate the outcomes of vaginal mesh surgery.

In summary, the authors stated that in $23 \%$ of women, a mesh-related complication occurred. The domain scores of the four questionnaires showed no statistical difference between women with and without a mesh-related complication. The authors concluded that when counseling patients about adverse events relating to vaginal mesh surgery, women should be informed that mesh-related complications do not negatively affect QoL related to micturition, defecation, and sexual functioning.

The authors present an interesting study in light of the recent issues pertaining to vaginal mesh surgery. Limitations of the study include the response rate of only $68 \%$. Also of note, the revised PISQ version (PISQ-IR) would have been a better choice of questionnaire, as it allows for measurement of sexual well-being in women who are not sexually active. The authors state that the comparison of PISQ scores between women with and without a mesh-related complication showed no difference. Importantly, the groups were small for an adequate comparison. Additionally, the authors also highlight a relationship between frequency of sexual activity and mesh erosion rate. However, given the small sample sizes, this may merely be a small number error.

\section{Compliance with ethical standards}

Conflicts of interest None.

Publisher's note Springer Nature remains neutral with regard to jurisdictional claims in published maps and institutional affiliations.

Abdelmageed Abdelrahman

abdelmageed@hotmail.co.uk

1 Department of Urogynaecology, Liverpool Women's Hospital NHS Foundation Trust, Liverpool, UK 